\title{
A Simple FDTD Algorithm for Simulating EM-Wave Propagation in General Dispersive Anisotropic Material
}

\author{
Ahmad A. Al-Jabr, Student Member, IEEE, Mohammad A. Alsunaidi, Tien Khee, and \\ Boon S. Ooi, Senior Member, IEEE
}

\begin{abstract}
In this paper, an finite-difference time-domain (FDTD) algorithm for simulating propagation of EM waves in anisotropic material is presented. The algorithm is based on the auxiliary differential equation and the general polarization formulation. In anisotropic materials, electric fields are coupled and elements in the permittivity tensor are, in general, multiterm dispersive. The presented algorithm resolves the field coupling using a formulation based on electric polarizations. It also offers a simple procedure for the treatment of multiterm dispersion in the FDTD scheme. The algorithm is tested by simulating wave propagation in 1-D magnetized plasma showing excellent agreement with analytical solutions. Extension of the algorithm to multidimensional structures is straightforward. The presented algorithm is efficient and simple compared to other algorithms found in the literature.
\end{abstract}

Index Terms-Auxiliary differential equation finite-difference time domain (ADE FDTD), anisotropic, dispersive, gyrotropic.

\section{INTRODUCTION}

$\mathbf{T}$ HE finite-difference time-domain (FDTD) method is a leading numerical tool for modeling electromagnetic (EM) propagation in various materials ranging from ionoshperic plasmas [1] to gallium-nitrogen $(\mathrm{GaN})$ nanowires [2]. When applied to deal with anisotropic materials with tensorial permittivities, the simulation, however, is quite challenging because the electric fields are generally coupled. In particular, the simulation of anisotropic materials is challenging because the electric fields are generally coupled. The difficulty increases if the elements of the tensor are multiterm dispersive. Several researchers have proposed algorithms that deal efficiently with this kind of material. The first algorithm was proposed in 1992 by Hunsberger et al. which was based on the reclusive convolution (RC) approach [3]. The algorithm was valid for 1-D problems only. Later in 1993, Schneider pointed out potential inconsistencies in the Yee's mesh for anisotropic materials [4].

Manuscript received June 23, 2012; revised September 17, 2012; accepted October 16, 2012. Date of publication November 16, 2012; date of current version February 27, 2013.

A. A. Al-Jabr, T. K. Ng, and B. S. Ooi are with the Photonics Laboratory, Division of Physical Sciences and Engineering, King Abdullah University for Science and Technology (KAUST), Thuwal 23955-6900, Saudi Arabia (e-mail: boon.ooi@kaust.edu.sa).

M. A. Alsunaidi is with the Electrical Engineering Department, Photonics Group, King Fahd University of Petroeum and and Minerals (KFUPM), Dhahran 31261, Saudi Arabia (e-mail: msunaidi@kfupm.edu.sa).

Color versions of one or more of the figures in this paper are available online at http://ieeexplore.ieee.org.

Digital Object Identifier 10.1109/TAP.2012.2227447
He proposed a different arrangement of the fields to overcome this inconsistency. The algorithm presented by Hunsberger et al. was later used to study the propagation in rectangular waveguides filled with magnetized plasma [5], [6]. The first 3-D algorithm for anisotropic material was developed in 1999 [7]. This algorithm is a little bit complicated since it contains matrix multiplication in the FDTD loop and it is specifically built for magnetized plasma. Based on the JE convolution method (JEC), an algorithm is proposed and used to simulate propagation in magnetized plasma. The same validation example used by Hunsberger is used again [8]. The (JEC) was used for 2-D and 3-D study cases as found in [9] and [10]. The z-transform method was also used to develop an algorithm for anisotropic propagation and showed better accuracy over the RC method [11]. Consequently, the piecewise-linear RC method was introduced to improve the performance of the original RC algorithm [12]. Other methods were proposed to tackle the anisotropic propagation, including the shift operator FDTD [13], the Runge-Kutta exponential time differencing formulation (RKETD-FDTD) [14], EJ collocated method [15], and the exponential time differencing (ETD), FDTD method [16].

In this paper, a relatively simple multidimensional FDTD algorithm for the efficient and accurate simulation of anisotropic materials is proposed and tested. The algorithm is based on the auxiliary differential equation (ADE) approach where a general formulation of the electric polarization is used. In the following section, the formulations of the algorithm and the implementation procedure are introduced. In Section III, necessary verifications of the algorithm in a 1-D situation are provided. The extension of the algorithm to a 2-D example is given in Section IV.

\section{DERIVATION AND IMPLEMENTATION}

The electric flux density vector for a general anisotropic material can be written as

$$
D(w)=\left[\begin{array}{ccc}
\epsilon_{x x} & \epsilon_{x y} & \epsilon_{x z} \\
\epsilon_{y x} & \epsilon_{y y} & \epsilon_{y z} \\
\epsilon_{z x} & \epsilon_{z y} & \epsilon_{z z}
\end{array}\right]\left[\begin{array}{c}
E_{x}(w) \\
E_{y}(w) \\
E_{z}(w)
\end{array}\right] .
$$

For a nondispersive anisotropy, the discrete time equivalent of (1) is

$$
\begin{aligned}
& D_{x}^{n}=\epsilon_{x x} E_{x}^{n}+\epsilon_{x y} E_{y}^{n}+\epsilon_{x z} E_{z}^{n} \\
& D_{y}^{n}=\epsilon_{y x} E_{x}^{n}+\epsilon_{y y} E_{y}^{n}+\epsilon_{y z} E_{z}^{n} \\
& D_{z}^{n}=\epsilon_{z x} E_{x}^{n}+\epsilon_{z y} E_{y}^{n}+\epsilon_{z z} E_{z}^{n} .
\end{aligned}
$$


Using field placements according to the Yee's mesh, one can identify two problems. First, solving several dependent variables at the same instant of time is required. Second, locations of the electric-field components and flux density components are not consistent. These problems can be addressed by extending the general polarization algorithm reported recently in [17], which is based on the ADE approach introduced by Taflove [18]. Using this general algorithm, it is possible to decouple the fields in time efficiently. The main idea is to solve for these fields in two steps. First, polarization variables are appropriately introduced. Equation (2), for example, can be written as

$$
D_{x}^{n}=\epsilon_{0} E_{x}^{n}+P_{x x}^{n}+P_{x y}^{n}+P_{x z}^{n} .
$$

Second, polarizations are independently calculated using a linear combination of available polarization and field samples each with a weighting constant. For example (see derivation thereafter), a possible expression for $P_{x x}$ can be

$$
P_{x x}^{n}=C_{1} P_{x x}^{n-1}+C_{2} P_{x x}^{n-2}+C_{3} P_{x x}^{n-3}+C_{4} E_{x}^{n-1} .
$$

Similar equations are written for $P_{x y}^{n}$ and $P_{x z}^{n}$. It is to be noted that only previous time samples are needed. The constants $C_{1}$ to $C_{4}$ are calculated from the material dispersion relation. For the sake of demonstrating the applicability of the general algorithm and without loss of generality, the anisotropy associated with magnetized plasma is considered. Magnetized plasma is well studied in the literature and analytical expressions for reflection and transmission coefficients involving this material are available. The permittivity tensor for a magnetized plasma in the $\mathrm{z}$-direction is given by

$$
\epsilon_{i j}(w)=\left[\begin{array}{ccc}
\epsilon_{d}(w) & j \epsilon_{g}(w) & 0 \\
-j \epsilon_{g}(w) & \epsilon_{d}(w) & 0 \\
0 & 0 & \epsilon_{z}(w)
\end{array}\right]
$$

where the elements are defined as

$$
\begin{aligned}
\epsilon_{d}(w)=\epsilon_{0}\left[1-\frac{\left(\frac{w_{p}}{w}\right)^{2}\left[1-\left(\frac{j v_{c}}{w}\right)\right]}{\left[1-\left(\frac{j v_{c}}{w}\right)\right]^{2}-\left(\frac{w_{b}}{w}\right)^{2}}\right] \\
=\epsilon_{0}\left[1+\frac{w_{p}^{2}+\frac{v_{c} w_{p}^{2}}{j w}}{\left(v_{c}^{2}+w_{b}^{2}\right)+2 j v_{c} w-w^{2}}\right] \\
\epsilon_{g}(w)=\epsilon_{0}\left[\frac{\left(\frac{w_{p}}{w}\right)^{2}\left(\frac{w_{b}}{w}\right)}{\left[1-\left(\frac{j v_{c}}{w}\right)\right]^{2}-\left(\frac{w_{b}}{w}\right)^{2}}\right] \\
=\epsilon_{0}\left[\frac{-\frac{w_{b} w_{p}^{2}}{j w}}{\left(v_{c}^{2}+w_{b}^{2}\right)+2 j v_{c} w-w^{2}}\right] \\
\epsilon_{z}(w)=\epsilon_{0}\left[1-\frac{w_{p}^{2}}{w\left(w+j v_{c}\right)}\right] .
\end{aligned}
$$

In the above equations, $w_{p}$ is the plasma frequency measured in radians per second, $v_{c}$ is the electron collision rate in Hertz, and $w_{b}$ is the cyclotron frequency, which is related to the applied magnetic field. Now $D_{x}(w)$, for example, can be written as

$$
\begin{array}{r}
D_{x}(w)=\epsilon_{0} E_{x}(w)+\epsilon_{0} \frac{w_{p}^{2}+\frac{v_{c} w_{p}^{2}}{j w}}{\left(v_{c}^{2}+w_{b}^{2}\right)+2 j v_{c} w-w^{2}} E_{x}(w) \\
+\epsilon_{0} \frac{-\frac{w_{b} w_{p}^{2}}{j w}}{\left(v_{c}^{2}+w_{b}^{2}\right)+2 j v_{c} w-w^{2}} E_{y}(w) .
\end{array}
$$

The second and the third terms can be replaced with the auxiliary variables $P_{x x}$ and $P_{x y}$, as

$$
D_{x}(w)=\epsilon_{0} E_{x}(w)+P_{x x}(w)+P_{x y}(w) .
$$

Taking the Fourier transform and then discretizing in time, (12) can be written in the format given by (5) as

$$
D_{x}^{n}=\epsilon_{0} E_{x}^{n}+P_{x x}^{n}+P_{x y}^{n} .
$$

The evaluation of $P_{x x}^{n}$ and $P_{x y}^{n}$ will be discussed shortly. The update equation for the electric field is then given by

$$
E_{x}^{n}=\frac{D_{x}^{n}-P_{x x}^{n}-P_{x y}^{n}}{\epsilon_{0}} .
$$

At the onset of calculations, all flux densities are evaluated from Maxwell's curl equation using present values of the magnetic-field intensity $H$. Because the dispersion relations representing the polarization variables $P_{x x}$ and $P_{x y}$ are not in the standard Lorentzian form [see (11)], a novel procedure will be devised. There are two ways of deriving the algorithm: the integral method and the derivative method.

\section{A. Integral Method}

For the integral method, we take the general dispersion relation form in (11) as

$$
P(w)=\frac{\frac{a_{-1}}{j w}+a_{0}}{b_{0}+b_{1} j w+b_{2} j w^{2}} E(w)
$$

where $a_{-1}, a_{0}, b_{0}, b_{1}$, and $b_{2}$ are just numerical coefficients and their subscripts refer to the power of $(j w)$. These coefficients determine the material behavior. By setting some of them to zero, it is possible to produce other dispersion forms. Multiplying (15) by the denominator and applying Fourier transform gives

$$
b_{0} P+b_{1} P^{\prime}+b_{2} P^{\prime \prime}=a_{-1} \int E+a_{0} E .
$$

Discretizing and approximating derivatives in the time domain around the time step $(n-1)$, one obtains

$$
\begin{aligned}
b_{0} P^{n-1}+b_{1} \frac{P^{n}-P^{n-2}}{2 \Delta t} & +b_{2} \frac{P^{n}-2 P^{n-1}+P^{n-2}}{\Delta t^{2}} \\
& =\Delta a_{-1} \sum_{k=0}^{n-1} E^{k}+a_{0} E^{n-1} .
\end{aligned}
$$


The update equation for the polarization variable is

$$
\begin{aligned}
P^{n}= & \frac{4 b_{2}-2 \Delta t^{2} b_{0}}{\Delta t b_{1}+2 b_{2}} P^{n-1}+\frac{\Delta t b_{1}-2 b_{2}}{\Delta t b_{1}+2 b_{2}} P^{n-2} \\
& \frac{2 \Delta t^{2} a_{0}}{\Delta t b_{1}+2 b_{2}} E^{n-1}+\frac{2 a_{-1} \Delta t^{3}}{\Delta t b_{1}+2 b_{2}} \sum_{k=0}^{n-1} E^{k} .
\end{aligned}
$$

\section{B. Derivative Method}

For the derivative method, the dispersion function in (11) is rewritten as

$$
\begin{aligned}
D_{x}(w)= & \epsilon_{0} E_{x}(w)+\epsilon_{0} \frac{w_{p}^{2}}{\left(v_{c}^{2}+w_{b}^{2}\right)+2 j v_{c} w-w^{2}} E_{x}(w) \\
& +\epsilon_{0} \frac{v_{c} w_{p}^{2}}{j w\left(v_{c}^{2}+w_{b}^{2}\right)-2 v_{c} w^{2}-j w^{3}} E_{x}(w) \\
& +\epsilon_{0} \frac{-w_{b} w_{p}^{2}}{j w\left(v_{c}^{2}+w_{b}^{2}\right)-2 v_{c} w^{2}-j w^{3}} E_{y}(w)
\end{aligned}
$$

to eliminate the integrals. The third and fourth terms are not standard Lorentzians, and so they are treated as follows. The polarization in this case is written as

$$
P(w)=\frac{a_{0}}{b_{0}+b_{1} j w+b_{2}(j w)^{2}+b_{3}(j w)^{3}} E(w) .
$$

Using a similar procedure as in the integral method, one obtains

$$
b_{0} P(t)+b_{1} P^{\prime}(t)+b_{2} P^{\prime \prime}(t)+b_{3} P^{\prime \prime \prime}(t)=a_{0} E(t) .
$$

Discretizing the equation around $(n-1)$, we obtain

$$
\begin{array}{r}
b_{0} P^{n-1}+b_{1} \frac{P^{n}-P^{n-2}}{2 \Delta t}+b_{2} \frac{P^{n}-2 P^{n-1}+P^{n-2}}{\Delta t^{2}}+ \\
b_{3} \frac{P^{n}-3 P^{n-1}+3 P^{n-2}-P^{n-3}}{\Delta t^{3}}=a_{0} E^{n-1} .
\end{array}
$$

Finally, the update equation reads

$$
\begin{aligned}
P^{n}= & \frac{4 b_{2}-2 \Delta t^{2} b_{0}+6 \frac{b_{3}}{\Delta t}}{\Delta t b_{1}+2 b_{2}+2 \frac{b_{3}}{\Delta t}} P^{n-1}+\frac{\Delta t b_{1}-2 b_{2}-6 \frac{b_{3}}{\Delta t}}{\Delta t b_{1}+2 b_{2}+2 \frac{b_{3}}{\Delta t}} P^{n-2} \\
& +\frac{\frac{b_{3}}{\Delta t}}{\Delta t b_{1}+2 b_{2}+2 \frac{b_{3}}{\Delta t}} P^{n-3}+\frac{2 \Delta t^{2} a_{0}}{\Delta t b_{1}+2 b_{2}+2 \frac{b_{3}}{\Delta t}} E^{n-1} .
\end{aligned}
$$

It should be remarked that at (22), the first and second derivatives are centered around the $(n-1)$ time step while the third derivative is not. Therefore, this method is expected to give less accuracy than the integral method. With these derivations, we have solved the problem of time coupling. For field component placement, it has been found that instead of the corrections to Yee's mesh proposed in [4], averaging the electric fields in space around the point of calculation can solve the problem of inconsistency. The electric fields in (18) and (23) need to be corrected by using averages calculated from neighboring spatial points as

$$
\bar{E}_{i}=\frac{E_{i-1}+E_{i+1}}{2}
$$

for a 1-D case, where $i$ represents the space index.

\section{VERIFICATIONS}

For verification, the standard 1-D reflection/transmission test is considered [3]. Propagation is taken in the z-direction and all $\partial / \partial x$ and $\partial / \partial y$ are removed. Therefore, the simulated fields are $E_{x}, H_{y}, E_{y}$, and $H_{x}$. Half of the space is air and the other half is made of magnetized plasma. A Gaussian pulse is launched in air with a waist of $1 \mathrm{ps}$. Only $E_{x}$ and $H_{y}$ are excited in the air region. However, once the wave reaches the interface, $E_{y}$ and $H_{x}$ inside the magnetized plasma will start to evolve because of the anisotropy. The reflected and transmitted fields are recorded in time. The frequency spectrum is calculated using Fourier transform and then normalized to the excited pulse. The right circularly polarized (RCP) and left circularly polarized (LCP) reflection and transmission coefficients are calculated using the following equations [3]:

$$
\begin{aligned}
& T=E_{x t}(w) \pm j E_{y t}(w),(+R /-L) \\
& \Gamma=E_{x r}(w) \pm j E_{y r}(w),(+R /-L) .
\end{aligned}
$$

The fields $E_{x r}(w)$ and $E_{x t}(w)$ refer to the reflected and transmitted $E_{x}$ in the frequency domain after normalization. The analytical values of the coefficients are calculated using the relations

$$
\begin{aligned}
& \Gamma=\frac{\sqrt{\epsilon_{\mathrm{eff}}}-\sqrt{\epsilon_{0}}}{\sqrt{\epsilon_{\mathrm{eff}}}+\sqrt{\epsilon_{0}}} \\
& T=\frac{2 \sqrt{\epsilon_{\mathrm{eff}}}}{\sqrt{\epsilon_{\mathrm{eff}}}+\sqrt{\epsilon_{0}}} .
\end{aligned}
$$

The effective permittivity $\epsilon_{e f f}$ for RCP and LCP is given as

$$
\epsilon_{e f f}(w)=\epsilon_{d}(w) \pm \epsilon_{g}(w),(+L /-R)
$$

which can be written as

$$
\epsilon_{e f f}(w)=\epsilon_{0}\left[1+\frac{w_{p}^{2}}{w\left[\left(j v_{c} \pm w\right)+w\right]}\right],(-L /+R) .
$$

The parameters of the magnetized plasma are taken as $w_{p}=$ $2 \pi \times 50 \times 10^{9} \mathrm{rad} / \mathrm{s}, v_{c}=2 \times 10^{10} \mathrm{~Hz}$, and $w_{b}=3 \times$ $10^{11} \mathrm{rad} / \mathrm{s}$. For the simulated transmission coefficient, the fields are recorded at a distance $d$ from the interface in the plasma region. Therefore, the simulated transmission coefficient given by (26) is modified as

$$
T=\exp ^{-j \beta d}\left[E_{x t}(w) \pm j E_{y t}(w)\right],(-L /+R)
$$

where $\beta=w \sqrt{\mu_{0} \epsilon_{e f f}}$ is the phase constant. The integral method is used with a discretization distance $\Delta z$ of $75 \mu \mathrm{m}$. Reflection and transmission coefficients from the simulation are shown in Fig. 1, where excellent agreement with analytical results is achieved. The resonance and cutoff frequencies are defined as the frequencies that make $\beta$ equal to infinity and zero, respectively. Referring back to (30), resonance is easily found at $w=j v_{c} \pm w_{b}$. Similarly, the cutoff can be found by solving for $w$. For LCP, the resonance and cutoff frequencies are 47.9 and $79.3 \mathrm{GHz}$, respectively. For RCP, the cutoff frequency is $31.5 \mathrm{GHz}$. 

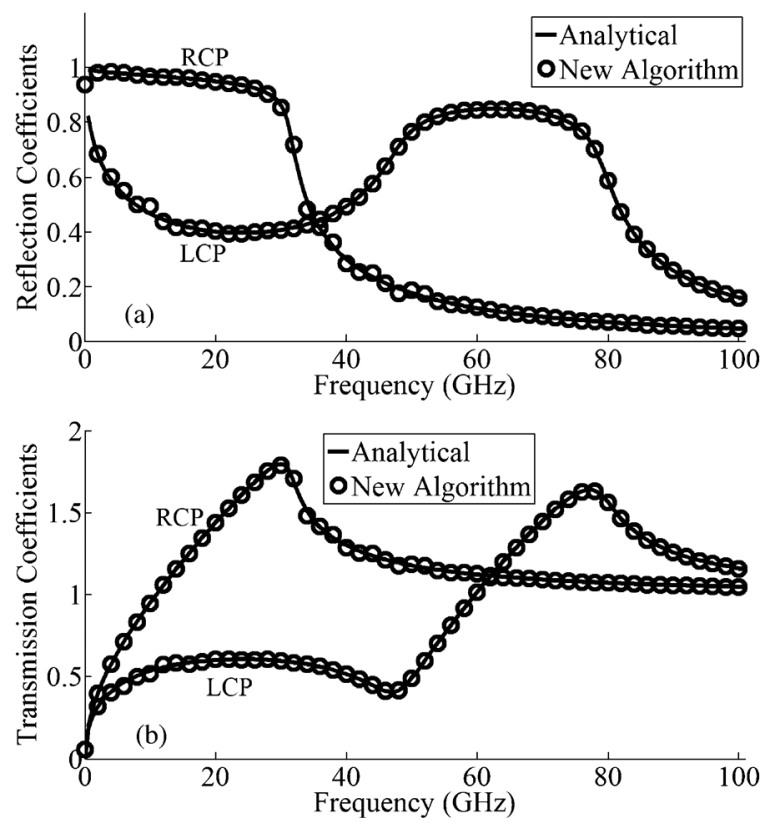

Fig. 1. Simulated and analytical reflection and transmission coefficients at normal incidence of a plane wave hitting the air-magnetized plasma interface: (a) reflection coefficient and (b) transmission coefficient.

The problem of the magnetized plasma slab presented in [3] is also considered. A plane wave impinges on a 9-mm slab of magnetized plasma having the same parameters as in the previous example. We use a discretization distance of $\Delta z$ of $75 \mu \mathrm{m}$ and a total simulation time enough for the fields to settle down to zero. PML is applied to truncate the simulation space. The analytical expressions for the reflection and transmission coefficients are calculated as in [19] using the effective permittivity

$$
\begin{aligned}
\Gamma & =\frac{\Gamma_{12}+\Gamma_{23} e^{-j 2 \beta d}}{1+\Gamma_{12} \Gamma_{23} e^{-j 2 \beta d}} \\
T & =\frac{T_{12} T_{23} e^{-j 2 \beta d}}{1+T_{12} T_{23} e^{-j 2 \beta d}} .
\end{aligned}
$$

Here, $d$ is the thickness of the slab. Again, for the simulated transmission coefficient, a correction factor similar to the one in (31) is used. Figs. 2 and 3 show excellent agreement between simulated and analytical coefficients with an average absolute error $|\Delta|<0.006$.

The main advantages of this new algorithm are summarized in the following text. It is obvious from (2)-(4) that the field components are coupled. The efficiency of this algorithm lies in the fact that all polarizations are decoupled and can be independently calculated outright for the whole domain. Subsequently, such calculations can be pulled out of the main field loops. Also, the number of operations in each time step is substantially less than the reported algorithms, especially for 3-D simulations. The algorithm is robust in the sense that any frequency-dependent dispersion relation and any arrangements of the permittivity tensor elements can be handled within the mainframe described earlier with the problem reduced to the evaluation of four constants.
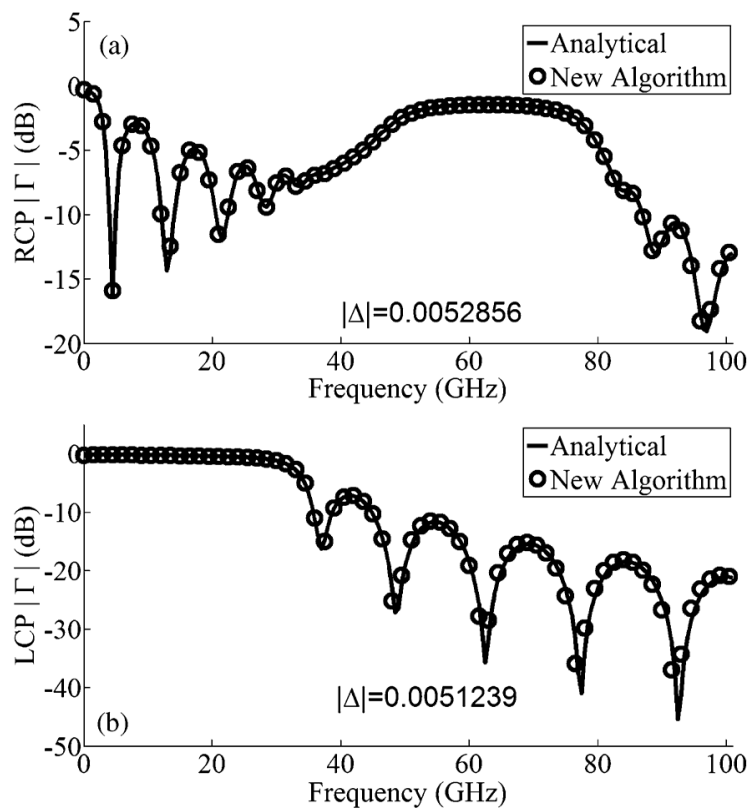

Fig. 2. Simulated and analytical reflection coefficients at normal incidence of a plane wave hitting a 9-mm slab of magnetized plasma: (a) RCP and (b) LCP reflection coefficients.
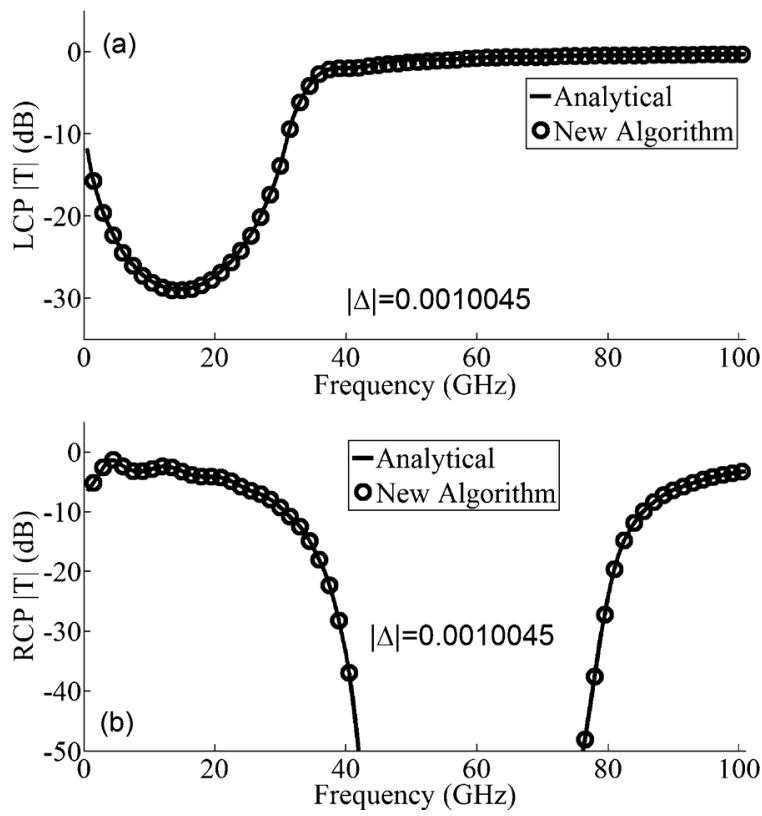

Fig. 3. Simulated and analytical transmission coefficients at normal incidence of a plane wave hitting a 9-mm slab of magnetized plasma: (a) LCP and (b) RCP transmission coefficients.

\section{2-D SiMULATION}

The algorithm is extended and tested on a 2-D problem. Because the algorithm decouples the fields very easily, the extension from 1-D to 2-D is straightforward. To test its applicability, the propagation of a TE-polarized plane wave is examined. A $y$-polarized incident field propagates in the $x$-direction, and impinges on a circular disk of plasma with a diameter of $10 \mathrm{~mm}$. The whole calculation space is $20 \times 20 \mathrm{~mm}$ in size with $\Delta x=$ $\Delta y=100 \mu \mathrm{m}$. PML-absorbing boundaries are applied at the 

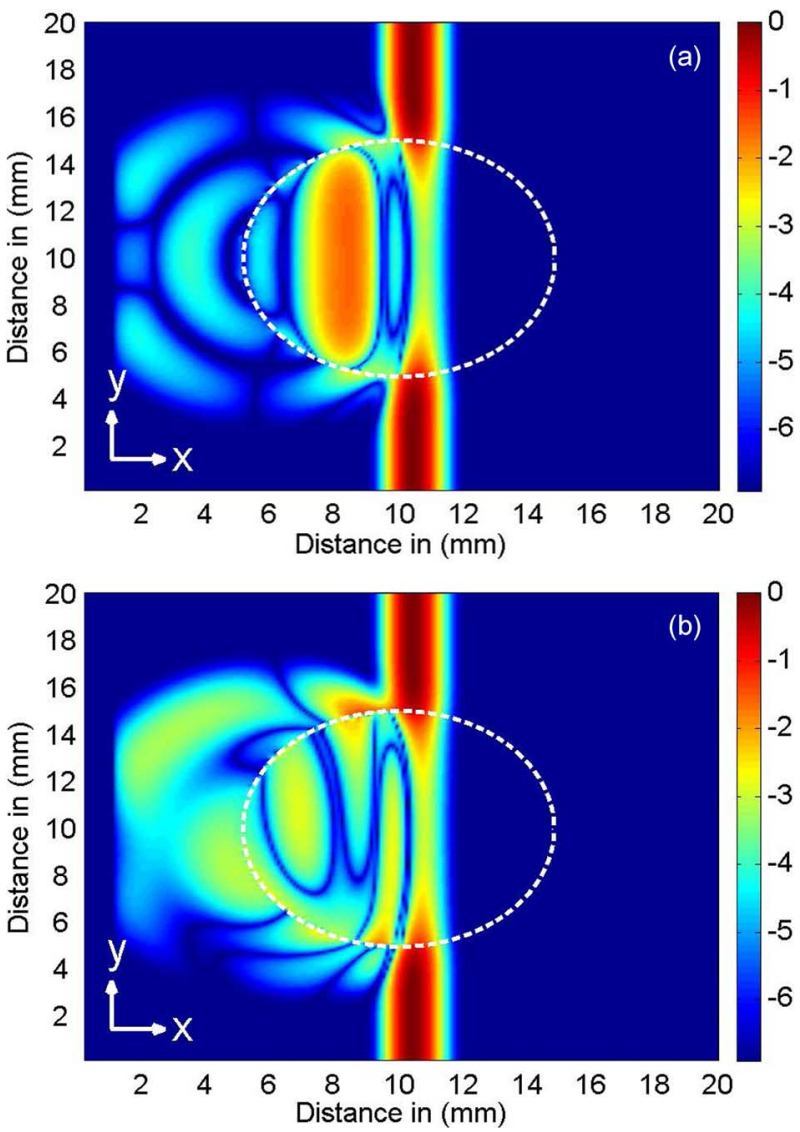

Fig. 4. Plane wave impinging on a circular disk of plasma. (a) Snapshot of Hertz (in decibels) in isotropic plasma. (b) Snapshot of Hertz (in decibels) in magnetized plasma.

borders to truncate the simulation domain. In the first simulation, isotropic plasma is used where all diagonal elements have the same form of $\epsilon_{z}(w)$ as given in (10) with numerical values used in the verification section. A snapshot of the simulation is shown in Fig. 4(a), where the Hertz field, which is perpendicular to the page, is plotted. As expected, the propagation is typical of propagation in isotropic media where the symmetry of the propagation of the incident wave within the disk is preserved, noting that the excitation source profile and the material structure are both spatially symmetric. In the second simulation, a magnetic field is applied to the plasma converting the permittivity tensor to the gyrotropic tensor with the same numerical values given in the verification section. The Hertz field is plotted again at the same instant of time. As shown in Fig. 4(b), the propagation behavior is different due to material anisotropy, where the existence of a preferred propagation direction is clearly demonstrated. The purpose of this example is just to show that the presented algorithm is extendable from 1-D to 2-D without extra derivation.

\section{CONCLUSION}

A simple FDTD algorithm for the simulation of general dispersive anisotropic media is derived and tested. It is based on the ADE approach and the generalized polarization formulation. The algorithm can be easily extended to 3-D structures. Compared to previously reported schemes, this algorithm is efficient and robust. The utilization of this algorithm in the simulation of ferrite material is straightforward.

\section{REFERENCES}

[1] Y. Yu, J. Niu, and J. Simpson, “A 3-D global earth-ionosphere FDTD model including an anisotropic magnetized plasma ionosphere," IEEE Trans. Antennas Propag., vol. 60, no. 7, pp. 3246-3256, Jul. 2012.

[2] A. Maslov and C. Ning, "Modal gain in a semiconductor nanowire laser with anisotropic bandstructure," IEEE J. Quantum Electron., vol. 40, no. 10 , pp. 1389-1397, Oct. 2004.

[3] F. Hunsberger, R. Luebbers, and K. Kunz, "Finite-difference time-domain analysis of gyrotropic media. I. Magnetized plasma," IEEE Trans. Antennas Propag., vol. 40, no. 12, pp. 1489-1495, Dec. 1992.

[4] J. Schneider and S. Hudson, "A finite-difference time-domain method applied to anisotropic material," IEEE Trans. Antennas Propag., vol. 41, no. 7, pp. 994-999, Jul. 1993.

[5] M. Segatto, R. Goncalves Farias, and A. Giarola, "Electromagnetic wave propagation in waveguides with magnetized plasma," in Proc. Antennas Propag. Soc. Int. Symp., 1995, vol. 1, pp. 652-655.

[6] H. Muta, T. Sakoda, Y. Ueda, and Y. Kawai, "One-dimensional simulation of microwave propagation in electron cyclotron resonance plasmas," Jpn. J. Appl. Phys., vol. 36, pt. 1, pp. 872-876, 1997.

[7] J. Lee and D. Kalluri, "Three-dimensional FDTD simulation of electromagnetic wave transformation in a dynamic inhomogeneous magnetized plasma," IEEE Trans. Antennas Propag., vol. 47, no. 7, pp. 1146-1151, Jul. 1999.

[8] S. Lui, J. Mo, and N. Yuan, "FDTD analysis of electromagnetic reflection by conductive plane covered with magnetized inhomogeneous plasmas," Int. J. Infrared Millimeter Waves, vol. 23, no. 12, pp. $1803-1815,2002$.

[9] L. Xu and N. Yuan, "JEC-FDTD for 2-D conducting cylinder coated by anisotropic magnetized plasma," IEEE Microw. Wireless Compon. Lett., vol. 15 , no. 12 , pp. 892-894, Dec. 2005.

[10] L. Xu and N. Yuan, "FDTD formulations for scattering from 3-D anisotropic magnetized plasma objects," IEEE Antennas Wireless Propag. Lett., vol. 5, no. 1, pp. 335-338, Dec. 2006.

[11] S. Huang and F. Li, "FDTD simulation of electromagnetic propagation in magnetized plasma using z transforms," Int. J. Infrared Millimeter Waves, vol. 25, no. 5, pp. 815-825, 2004

[12] S. Liu, J. Mo, and N. Yuan, "Piecewise linear current density recursive convolution FDTD implementation for anisotropic magnetized plasmas," IEEE Microw. Wireless Compon. Lett., vol. 14, no. 5, pp. 222-224, May 2004.

[13] H. Yang, R. Chen, and Y. Zhou, "SO-FDTD analysis on magnetized plasma," Int. J. Infrared Millimeter Waves, vol. 28, no. 9, pp. 751-758, 2007.

[14] S. Liu and S. Liu, "Runge-Kutta exponential time differencing FDTD method for anisotropic magnetized plasma," IEEE Antennas Wireless Propag. Lett., vol. 7, pp. 306-309, 2008.

[15] Y. Yu and J. Simpson, "An EJ collocated 3-D FDTD model of electromagnetic wave propagation in magnetized cold plasma," IEEE Trans. Antennas Propag., vol. 58, no. 2, pp. 469-478, Feb. 2010.

[16] S. Huang and F. Li, "FDTD implementation for magnetoplasma medium using exponential time differencing," IEEE Microw. Wireless Compon. Lett., vol. 15, no. 3, pp. 183-185, Mar. 2005.

[17] M. Alsunaidi and A. Al-Jabr, "A general ADE-FDTD algorithm for the simulation of dispersive structures," IEEE Photon. Technol. Lett., vol. 21, no. 12, pp. 817-819, Jun. 15, 2009.

[18] A. Taflove and S. Hagness, Computational Electrodynamics. Norwood, MA: Artech House, 1995.

[19] C. Balanis, Advanced Engineering Electromagnetics. New York: Wiley, 1989

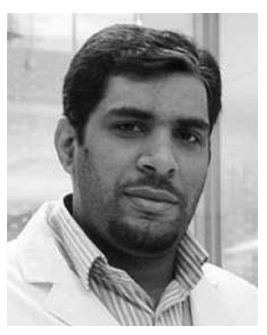

Ahmad A. Al-Jabr (S'11) received the B.Sc. and M.Sc. degrees in electrical engineering from King Fahd University of Petroleum and Minerals (KFUPM), Dhahran, Saudi Arabia.

He was a Lecturer at Jubail Industrial College. In 2010, he joined King Abdullah University for Science and Technology, Thuwal, Saudi Arabia, as a Ph.D. student in the photonics lab. His research includes modeling of semiconductor lasers and nanostructures in finite-difference time domain.

Mr. Al-Jabr is a student member of OSA.

Mohammad A. Alsunaidi, photograph and biography not available at the time of publication. 


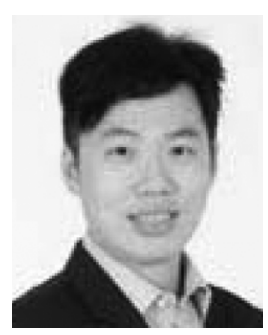

Tien Khee received the Ph.D. degree in electrical and electronics engineering from Nanyang Technological University (NTU), Singapore, researching molecular-beam epitaxy (MBE)-grown dilute-nitride quantum well structures for optical telecommunication applications.

$\mathrm{He}$ is currently researching III-V-based quantum structures for light-emitting applications at King Abdullah University for Science and Technology, Thuwal, Saudi Arabia.. His M.Eng. research at the same laboratory focused on electron cyclotron resonance plasma etching of As-based and P-based III-V semiconductors for eventual application in heterojunction bipolar transistors. In 2007, he accepted a Research Fellow position at NTU, conducting research into MBE-grown gallium-arsenide nanowires, quantum-dots structures, and dilute-nitride-antimonide solar cells using silicon-germanium- and silicon-based heterogeneous substrates. Previously, he was an Engineer for Tinggi Technologies, Singapore, in 2004 , a startup company that designed and fabricated high-power gallium-nitrogen blue light-emitting diodes on highly conductive substrates. He later worked as a member of the technical staff and as a Thin Film Process Manager in the same company.

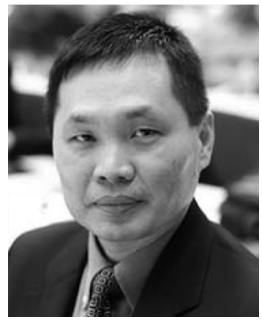

Boon S. Ooi (M'95-SM'03) received the Ph.D. degree in electronics and electrical engineering from the University of Glasgow, Glasgow, U.K.

$\mathrm{He}$ served as a faculty member at Nanyang Technological University, Singapore, and Lehigh University, Bethlehem, PA, USA, before joining King Abdullah University for Science and Technology, Thuwal, Saudi Arabia, as a Professor of Electrical Engineering in 2009. His research interests include semiconductor lasers and photonics integrated circuits.

Prof. Ooi is a Fellow of the SPIE and the Institute of Physics (U.K.) 